\title{
A Characterization of Strong Completeness in Fuzzy Metric Spaces
}

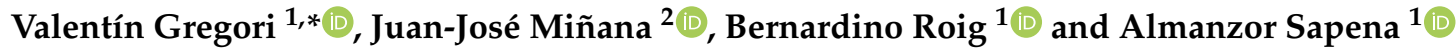 \\ 1 Instituto de Investigación para la Gestión Integrada de Zonas Costeras, Universitat Politècnica de València, \\ C/ Paranimf, 1, 46730 Grao de Gandia, Spain; broig@mat.upv.es (B.R.); alsapie@mat.upv.es (A.S.) \\ 2 Departament de Ciències Matemàtiques i Informàtica, Universitat de les Illes Balears, \\ Carretera de Valldemossa km. 7.5, 07122 Palma, Spain; jj-minana@uib.es \\ * Correspondence: vgregori@mat.upv.es
}

Received: 29 April 2020; Accepted: 21 May 2020; Published: 26 May 2020

\begin{abstract}
Here, we deal with the concept of fuzzy metric space $(\mathcal{X}, \mathcal{M}, *)$, due to George and Veeramani. Based on the fuzzy diameter for a subset of $\mathcal{X}$, we introduce the notion of strong fuzzy diameter zero for a family of subsets. Then, we characterize nested sequences of subsets having strong fuzzy diameter zero using their fuzzy diameter. Examples of sequences of subsets which do or do not have strong fuzzy diameter zero are provided. Our main result is the following characterization: a fuzzy metric space is strongly complete if and only if every nested sequence of close subsets which has strong fuzzy diameter zero has a singleton intersection. Moreover, the standard fuzzy metric is studied as a particular case. Finally, this work points out a route of research in fuzzy fixed point theory.
\end{abstract}

Keywords: fuzzy metric; Cauchy sequence; (strong) convergence; completeness; fuzzy diameter

MSC: 54A40; 54D35; 54E50

\section{Introduction}

Kramosil and Michalek [1] extended the concept of Menger space [2] to fuzzy setting. Here, we deal with the concept of fuzzy metric due to George and Veeramani [3] which is a modification of the one given by Kramosil and Michalek. These concepts are formulated by means of a continuous $t$-norm $*$ [4]. If $(\mathcal{X}, \mathcal{M}, *)$ is a fuzzy metric space, then $\mathcal{M}$ is a fuzzy metric on $\mathcal{X}$ that induces a topology $\tau_{\mathcal{M}}$ on $\mathcal{X}$. Gregori and Romaguera [5] proved that $\tau_{\mathcal{M}}$ is metrizable. Then, many topics studied in our fuzzy setting are extensions of the corresponding ones in classical metrics. Now, a significant difference with classical metrics is that fuzzy metrics do not admit, in general, (fuzzy) completion [6]. On the other hand, fuzzy metrics were extended to the intuitionistic context introduced by Atanassov [7] (see, for instance, [8-10]). An area of high activity in our context is fuzzy fixed point theory [11-13]. In this context, as in the classical case, the concept of Cauchy sequence plays a crucial role. Several concepts of Cauchy sequence in this context have been given in the literature [14]. In particular, and in a natural way, Gregori and Miñana [15] introduced and studied a concept of Cauchy sequence, named strong Cauchy sequence (Definition 4). Nevertheless, up to now, the two more used concepts of Cauchy sequence in fuzzy fixed point theory (in a wide sense) are due to M. Grabiec [16] and George and Veeramani [17]. The first one, denoted here G-Cauchy, was introduced for fuzzy metrics in the sense of Kramosil and Michalek (a sequence $\left\{\xi_{n}\right\}_{n \in \mathbb{N}}$ is $G$-Cauchy if $\lim _{n} M\left(\xi_{n}, \xi_{n+1}, t\right)=1$ for all $t>0$, as observed Mihed in [18]). The given one by George and Veeramani is simply called Cauchy (Definition 2). A discussion on these two concepts can be seen in [19]. If we assume for each one of the three Cauchy concepts above mentioned, the concept, as usual, of completeness (see Definition 9), we have the following chain of implications, of obvious interpretation, for a fuzzy metric space. 


$$
\text { G-complete } \rightarrow \text { complete } \rightarrow \text { strongly complete }
$$

The aim of this paper is to study the concept of (strong) fuzzy diameter zero for a family of sets of $\mathcal{X}$, and then, to find a characterization of the strong completeness of $\mathcal{X}$, similar to the classical one, by means of certain family of nested sequences of sets of $\mathcal{X}$. For it, we proceed as explained, briefly, in the following paragraph.

Inspired by George and Veeramani [17] (Definition 3.1), and accordingly to the concepts introduced in [15], we introduce the concept of a family of subsets which has strong fuzzy diameter zero. In particular, a nested sequence of non-empty sets $\left\{\mathcal{A}_{n}\right\}_{n \in \mathbb{N}}$ of $\mathcal{X}$ has strong fuzzy diameter zero if given $r \in] 0,1$ [ there exists $n_{r} \in \mathbb{N}$, depending on $r$, such that $\mathcal{M}(\xi, \eta, t)>1-r$ for each $\xi, \eta \in \mathcal{A}_{n}$ with $n \geq n_{r}$ and all $t \in \mathbb{R}^{+}$(Remark 4). Then, using the notion of fuzzy diameter $\phi_{\mathcal{A}}(t)$ of a subset $\mathcal{A}$ of $\mathcal{X}$ (Definition 6), we characterize, in Proposition 3, those nested sequences which have strong fuzzy diameter zero. The existence of (non-trivial) nested sequences which have strong fuzzy diameter zero, is shown, among other things, in Example 1. In the proof of Lemma 1, we show that $\mathcal{A}$ and $\overline{\mathcal{A}}$ (closure of $\mathcal{A}$ ) have the same fuzzy diameter. Then, we use Lemma 1 to establish, in Theorem 1, our main result: $(\mathcal{X}, \mathcal{M}, *)$ is strongly complete if and only if every nested sequences of non-empty closed sets of $\left(\mathcal{X}, \tau_{\mathcal{M}}\right)$ with strong fuzzy diameter zero, has a singleton intersection. As a corollary, we obtain that every standard fuzzy metric space is strongly complete (Proposition 7). As application, again of Theorem 1, we prove in Example 3 the existence of a (non-trivial) strongly complete fuzzy metric space, previously studied in Example 2. Finally, recalling the above chain of implications between the different types of completeness, we point out the interest of the strong completeness in order to generalize fixed point theorems stated for complete fuzzy metric spaces.

The structure of the paper is as follows. In Section 2, we give known concepts and results that are needed for the comprehension of the paper. In Section 3, we introduce and study the concept of strong fuzzy diameter zero for a nested sequence of sets of $\mathcal{X}$. In Section 4 , we study some aspects related to the strong completeness and characterize when $(\mathcal{X}, \mathcal{M}, *)$ is strongly complete. Section 5 is an explanatory note on the possibility of continuing this work and also on the capability of using strong completeness in fuzzy fixed point theory.

\section{Preliminaries}

Throughout the paper, $\mathbb{R}^{+}$denotes the set of positive real numbers, i.e., $\left.\mathbb{R}^{+}=\right] 0, \infty[$.

Definition 1 ([3]). A fuzzy metric space is an ordered triple $(\mathcal{X}, \mathcal{M}, *)$ such that $\mathcal{X}$ is a (non-empty) set, $*$ is a continuous t-norm, and $\mathcal{M}$ is a fuzzy set on $\mathcal{X} \times \mathcal{X} \times \mathbb{R}^{+}$satisfying the following conditions, for all $\xi, \eta, \theta \in \mathcal{X}$ and $t, s \in \mathbb{R}^{+}$

$(\mathrm{GV} 1) \mathcal{M}(\xi, \eta, t)>0$;

(GV2) $\mathcal{M}(\xi, \eta, t)=1$ if and only if $\xi=\eta$;

(GV3) $\mathcal{M}(\xi, \eta, t)=\mathcal{M}(\eta, \xi, t)$;

$(\mathrm{GV} 4) \mathcal{M}(\xi, \eta, t) * \mathcal{M}(\eta, \theta, s) \leq \mathcal{M}(\xi, \theta, t+s)$; and

(GV5) $\left.\left.\mathcal{M}(\xi, \eta,-): \mathbb{R}^{+} \rightarrow\right] 0,1\right]$ is continuous.

If $(\mathcal{X}, \mathcal{M}, *)$ is a fuzzy metric space, we say that $(\mathcal{M}, *)$, or simply $\mathcal{M}$, is a fuzzy metric on $\mathcal{X}$. In addition, we say that $(\mathcal{X}, \mathcal{M})$ or, simply, $\mathcal{X}$ is a fuzzy metric space.

It is well known that the function $\mathcal{M}_{\xi, \eta}(t)=\mathcal{M}(\xi, \eta, t)$ for all $t>0$, is a non-decreasing function on $t$. When $\mathcal{M}(\xi, \eta, t)$ does not depend on $t$, that is $\mathcal{M}_{\xi, \eta}$ is constant, $\mathcal{M}$ is called stationary [20].

Let $(\mathcal{X}, d)$ be a metric space. Denote by $\cdot$ the usual product on $[0,1]$, and let $\mathcal{M}_{d}$ be the fuzzy set defined on $\mathcal{X} \times \mathcal{X} \times \mathbb{R}^{+}$by

$$
\mathcal{M}_{d}(\xi, \eta, t)=\frac{t}{t+d(\xi, \eta)} .
$$

Then, $\left(\mathcal{M}_{d}, \cdot\right)$ is a fuzzy metric on $\mathcal{X}$ called standard fuzzy metric induced by $d$ [3]. 
George and Veeramani proved [3] that every fuzzy metric $\mathcal{M}$ on $\mathcal{X}$ generates a topology $\tau_{\mathcal{M}}$ on $\mathcal{X}$ which has as a base the family of open sets of the form $\left\{\mathcal{B}_{\mathcal{M}}(\xi, r, t): \xi \in \mathcal{X}, r \in\right] 0,1\left[, t \in \mathbb{R}^{+}\right\}$, where $\mathcal{B}_{\mathcal{M}}(\xi, r, t)=\{\eta \in \mathcal{X}: \mathcal{M}(\xi, \eta, t)>1-r\}$ for all $\left.\xi \in \mathcal{X}, r \in\right] 0,1\left[\right.$ and $t \in \mathbb{R}^{+}$. In the case of the standard fuzzy metric $\mathcal{M}_{d}$, it is well known that the topology $\tau(d)$ on $\mathcal{X}$ deduced from $d$ satisfies $\tau(d)=\tau_{\mathcal{M}_{d}}$. From now on, we suppose $\mathcal{X}$ endowed with the topology $\tau_{\mathcal{M}}$.

As is usual, $\overline{\mathcal{A}}$ denotes the closure of the set $\mathcal{A}$ in $\mathcal{X}$ with respect to the topology $\tau_{\mathcal{M}}$.

Proposition 1 ([3]). A sequence $\left\{\xi_{n}\right\}_{n \in \mathbb{N}}$ in a fuzzy metric space $(\mathcal{X}, \mathcal{M}, *)$ converges to $\xi_{0}$ if and only if $\lim _{n} \mathcal{M}\left(\xi_{0}, \xi_{n}, t\right)=1$, for all $t \in \mathbb{R}^{+}$.

Definition 2 ([17]). A sequence $\left\{\xi_{n}\right\}_{n \in \mathbb{N}}$ in a fuzzy metric space $(\mathcal{X}, \mathcal{M}, *)$ is called Cauchy if for each $\varepsilon \in] 0,1\left[\right.$ and each $t \in \mathbb{R}^{+}$there exists $n_{0} \in \mathbb{N}$ such that $\mathcal{M}\left(\xi_{n}, \xi_{m}, t\right)>1-\varepsilon$ for all $n, m \geq n_{0}$ or equivalently $\lim _{m, n} \mathcal{M}\left(\xi_{n}, \xi_{m}, t\right)=1$ for all $t \in \mathbb{R}^{+}$.

$(\mathcal{X}, \mathcal{M}, *)$, or simply $\mathcal{M}$, is called complete if every Cauchy sequence in $\mathcal{X}$ is convergent with respect to $\tau_{\mathcal{M}}$.

Definition 3 ([15]). A sequence $\left\{\xi_{n}\right\}_{n \in \mathbb{N}}$ in a fuzzy metric space $(\mathcal{X}, \mathcal{M}, *)$ is called strongly convergent to $\xi_{0} \in \mathcal{X}$ if given $\left.\varepsilon \in\right] 0,1\left[\right.$ there exists $n_{\varepsilon}$, depending on $\varepsilon$, such that $\mathcal{M}\left(\xi_{n}, \xi_{0}, t\right)>1-\varepsilon$, for all $n \geq$ $n_{\varepsilon}$ and for all $t \in \mathbb{R}^{+}$.

Definition 4 ([15]). A sequence $\left\{\xi_{n}\right\}_{n \in \mathbb{N}}$ in a fuzzy metric space $(\mathcal{X}, \mathcal{M}, *)$ is strong Cauchy if given $\left.\varepsilon \in\right] 0,1[$ there exists $n_{\varepsilon}$, depending on $\varepsilon$, such that $M\left(\xi_{n}, \xi_{m}, t\right)>1-\varepsilon$, for all $n, m \geq n_{\varepsilon}$ and for all $t \in \mathbb{R}^{+}$.

\section{Fuzzy Diameter}

Along this paper $(\mathcal{X}, \mathcal{M}, *)$ is a fuzzy metric space, which we denote $(\mathcal{X}, \mathcal{M})$, or simply $\mathcal{X}$, if confusion is not possible.

Definition 5 ([21]). A non-empty set $\mathcal{A}$ of the fuzzy metric space $\mathcal{X}$ is said to be $\mathcal{F}$-bounded if there exist $r \in] 0,1\left[\right.$ and $t \in \mathbb{R}^{+}$such that $\mathcal{M}(\xi, \eta, t)>1-r$ for all $\xi, \eta \in \mathcal{A}$.

One can assume that this concept is appropriate, since as it is easy to verify that $\mathcal{A}$ is $\mathcal{F}$-bounded in a standard fuzzy metric $\left(\mathcal{X}, \mathcal{M}_{d}\right)$ if and only if $\mathcal{A}$ is bounded in the metric space $(\mathcal{X}, d)$.

In a metric space $(\mathcal{X}, d)$, the diameter of a non-empty set $\mathcal{A}$ of $\mathcal{X}$, denoted $\operatorname{diam}(\mathcal{A})$, is defined as $\operatorname{diam}(\mathcal{A})=\sup \{d(\xi, \eta): \xi, \eta \in \mathcal{A}\}$. In our fuzzy setting, diameter of $\mathcal{A}$ is defined as a function on the $t$-parameter, as follows:

Definition 6. The fuzzy diameter of a non-empty set $\mathcal{A}$ of a fuzzy metric space $\mathcal{X}$, with respect to $t$, is the function $\left.\phi_{\mathcal{A}}:\right] 0,+\infty[\rightarrow[0,1]$ given by

$$
\phi_{\mathcal{A}}(t)=\inf \{\mathcal{M}(\xi, \eta, t): \xi, \eta \in \mathcal{A}\}
$$

for each $t \in \mathbb{R}^{+}$.

Remark 1. The function $\phi_{\mathcal{A}}$ is, obviously, well defined and it is easy to observe:

(i) $\phi_{\mathcal{A}}(s) \leq \phi_{\mathcal{A}}(t)$ if $s<t$.

(ii) $\phi_{\mathcal{A}}(t) \geq \phi_{\mathcal{B}}(t)$ if $\mathcal{A} \subset \mathcal{B}$.

(iii) $\phi_{\mathcal{A}}(t)=1$ for some $t \in \mathbb{R}^{+}$if and only if $\mathcal{A}$ is a singleton set.

Definition 7 ([17]). A collection of sets $\left\{\mathcal{A}_{i}\right\}_{i \in \mathcal{I}}$ is said to have fuzzy diameter zero if given $\left.r \in\right] 0,1[$ and $t \in \mathbb{R}^{+}$there exists $i \in \mathcal{I}$ such that $\mathcal{M}(\xi, \eta, t)>1-r$ for all $\xi, \eta \in \mathcal{A}_{i}$. 
In the following, by a nested sequence of sets $\left\{\mathcal{A}_{n}\right\}_{n \in \mathbb{N}}$ we mean a sequence of non-empty sets $\mathcal{A}_{n}$ satisfying $\mathcal{A}_{n+1} \subset \mathcal{A}_{n}$ for all $n \in \mathbb{N}$.

Remark 2. In particular, a nested sequence of sets $\left\{\mathcal{A}_{n}\right\}_{n \in \mathbb{N}}$ has fuzzy diameter zero if for $\left.r \in\right] 0,1\left[\right.$ and $t \in \mathbb{R}^{+}$ there exists $n_{r, t} \in \mathbb{N}$ such that $\mathcal{M}(\xi, \eta, t)>1-r$ for each $\xi, \eta \in \mathcal{A}_{n}$ with $n \geq n_{r, t}$. In this case if $\bigcap_{n=1}^{\infty} \mathcal{A}_{n} \neq \varnothing$ then necessarily $\bigcap_{n=1}^{\infty} \mathcal{A}_{n}$ is a singleton set ([17], Remark 3.4).

Remark 3. Let $\left\{\mathcal{A}_{n}\right\}_{n \in \mathbb{N}}$ be a nested sequence of sets such that $\mathcal{A}_{n}=\mathcal{A}$ for $n \geq n_{0}$ (i.e. $\left\{\mathcal{A}_{n}\right\}_{n \in \mathbb{N}}$ is eventually constant). Then, $\left\{\mathcal{A}_{n}\right\}_{n \in \mathbb{N}}$ has fuzzy diameter zero if and only if $\mathcal{A}$ is a singleton set.

Roughly speaking, $\left\{\mathcal{A}_{n}\right\}_{n \in \mathbb{N}}$ has fuzzy diameter zero if, for each $t \in \mathbb{R}^{+}$, the sequence contains small sets whose (fuzzy) diameter tends to 1 . We formalize this in the following proposition.

Proposition 2. Let $\left\{\mathcal{A}_{n}\right\}_{n \in \mathbb{N}}$ be a nested sequence of sets of the fuzzy metric space $\mathcal{X}$. They are equivalent:

(i) $\left\{\mathcal{A}_{n}\right\}_{n \in \mathbb{N}}$ has fuzzy diameter zero.

(ii) $\lim _{n \rightarrow \infty} \phi_{\mathcal{A}_{n}}(t)=1$ for all $t \in \mathbb{R}^{+}$.

Proof. $(i) \rightarrow($ ii $)$ Let $t \in \mathbb{R}^{+}$. Given $\left.r \in\right] 0,1\left[\right.$ there exists $n_{r, t} \in \mathbb{N}$ such that $\mathcal{M}(\xi, \eta, t)>1-r$ for each $\xi, \eta \in \mathcal{A}_{n}$ with $n \geq n_{r, t}$. Then, $\phi_{\mathcal{A}_{n}}(t)=\inf \left\{\mathcal{M}(\xi, \eta, t): \xi, \eta \in \mathcal{A}_{n}\right\} \geq 1-r$ for all $n \geq n_{r, t}$. Hence $\lim _{n} \phi_{\mathcal{A}_{n}}(t)=1$, since $r$ is arbitrary in $] 0,1[$.

$($ ii $) \rightarrow(i)$ Suppose $\lim _{n \rightarrow \infty} \phi_{\mathcal{A}_{n}}(t)=1$ for all $t \in \mathbb{R}^{+}$. Let $t \in \mathbb{R}^{+}$and let $\left.r \in\right] 0,1[$. We can find $n_{r, t} \in \mathbb{N}$ such that $\phi_{\mathcal{A}_{n}}(t)>1-r$ for all $n \geq n_{r, t}$. Thus, $\mathcal{M}(\xi, \eta, t)>1-r$ for each $\xi, \eta \in \mathcal{A}_{n}$ with $n \geq n_{r, t}$ i.e., $\left\{\mathcal{A}_{n}\right\}_{n \in \mathbb{N}}$ has fuzzy diameter zero.

According to Definition 7, we introduce the following concept that is stronger than fuzzy diameter zero.

Definition 8. A family of non-empty sets $\left\{\mathcal{A}_{i}\right\}_{i \in \mathcal{I}}$ of $\mathcal{X}$ has strong fuzzy diameter zero if for $\left.r \in\right] 0,1[$ there exists $i \in \mathcal{I}$ such that $\mathcal{M}(\xi, \eta, t)>1-r$ for each $\xi, \eta \in \mathcal{A}_{i}$ and all $t \in \mathbb{R}^{+}$.

Remark 4. A nested sequence of sets $\left\{\mathcal{A}_{n}\right\}_{n \in \mathbb{N}}$ of $\mathcal{X}$ has strong fuzzy diameter zero if given $r \in[0,1[$ there exists $n_{r} \in \mathbb{N}$ such that $\mathcal{M}(\xi, \eta, t)>1-r$ for each $\xi, \eta \in \mathcal{A}_{n}$ with $n \geq n_{r}$ and all $t \in \mathbb{R}^{+}$.

Remark 5. If $\mathcal{M}$ is stationary both concepts in Definitions 7 and 8 agree.

Proposition 3. Let $(\mathcal{X}, \mathcal{M}, *)$ be a fuzzy metric space and let $\left\{\mathcal{A}_{n}\right\}_{n \in \mathbb{N}}$ be a nested sequence of sets of $\mathcal{X}$. They are equivalent:

(i) $\left\{\mathcal{A}_{n}\right\}_{n \in \mathbb{N}}$ has strong fuzzy diameter zero.

(ii) $\lim _{n \rightarrow \infty} \phi_{\mathcal{A}_{n}}\left(t_{n}\right)=1$ for every decreasing sequence of positive real numbers $\left\{t_{n}\right\}_{n \in \mathbb{N}}$ that converges to 0 .

Proof. $(i) \rightarrow(i i)$ Let $\left\{t_{n}\right\}_{n \in \mathbb{N}}$ be a decreasing sequence of positive real numbers that converges to 0 . Given $r \in] 0,1\left[\right.$, we can find $n_{r} \in \mathbb{N}$ such that $\mathcal{M}(\xi, \eta, t)>1-r$ for each $\xi, \eta \in \mathcal{A}_{n}$ with $n \geq n_{r}$ and all $t \in \mathbb{R}^{+}$. In particular, $\mathcal{M}\left(\xi, \eta, t_{n}\right)>1-r$ for all $\xi, \eta \in \mathcal{A}_{n}$ with $n \geq n_{r}$, i.e., $\phi_{\mathcal{A}_{n}}\left(t_{n}\right) \geq 1-r$ for all $n \geq n_{r}$, i.e., $\lim _{n \rightarrow \infty} \phi_{\mathcal{A}_{n}}\left(t_{n}\right)=1$.

$($ ii $) \rightarrow$ (i) Suppose that $\left\{\mathcal{A}_{n}\right\}_{n \in \mathbb{N}}$ has not strong fuzzy diameter zero. Let $\left.r \in\right] 0,1[$ such that $\mathcal{I}=\left\{n \in \mathbb{N}: \mathcal{M}(\xi, \eta, t) \leq 1-r\right.$, for some $\xi, \eta \in \mathcal{A}_{n}$ and some $\left.t \in \mathbb{R}^{+}\right\}$, is infinite. Take $n_{1}=\min \mathcal{I}$. Then, there exist $\xi_{n_{1}}, \eta_{n_{1}} \in \mathcal{A}_{n_{1}}$ such that $\mathcal{M}\left(\xi_{n_{1}}, \eta_{n_{1}}, t_{n_{1}}\right) \leq 1-r$ with $0<t_{n_{1}}<1$. 
Take $n_{2}>n_{1}$, with $n_{2} \in I$, such that $\mathcal{M}\left(\xi_{n_{2}}, \eta_{n_{2}}, t_{n_{2}}\right) \leq 1-r$ for some $\xi_{n_{2}}, \eta_{n_{2}} \in \mathcal{A}_{n_{2}}$ and $0<t_{n_{2}}<\min \left\{t_{n_{1}}, \frac{1}{2}\right\}$. In this way, we construct, by induction, a sequence $\left\{t_{n_{i}}\right\}_{i \in \mathbb{N}}$ such that $\mathcal{M}\left(\xi_{n_{i}}, \eta_{n_{i}}, t_{n_{i}}\right) \leq 1-r$ for some $\xi_{n_{i}}, \eta_{n_{i}} \in \mathcal{A}_{n_{i}}, n_{i} \in \mathcal{I}$ with $n_{i}>n_{i-1}$ and $0<t_{n_{i}}<\min \left\{t_{n_{i-1}}, \frac{1}{i}\right\}$.

Then, $\phi_{\mathcal{A}_{n_{i}}}\left(t_{n_{i}}\right)=\inf \left\{\mathcal{M}\left(\xi, \eta, t_{n_{i}}\right): \xi, \eta \in \mathcal{A}_{n_{i}}\right\} \leq \mathcal{M}\left(\xi_{n_{i}}, \eta_{n_{i}}, t_{n_{i}}\right) \leq 1-r$ for all $i \in \mathbb{N}$. Hence $\left\{\phi_{\mathcal{A}_{n_{i}}}\left(t_{n_{i}}\right)\right\}_{i \in \mathbb{N}}$ does not converge to 1 . Now, $\left\{t_{n_{i}}\right\}_{i \in \mathbb{N}}$ is a subsequence of the decreasing sequence $\left\{t_{n}\right\}_{n \in \mathbb{N}}$ that converges to 0 , given by $t_{n}=\left\{\begin{array}{ll}t_{n_{1}} & n \leq n_{1} \\ t_{n_{i+1}} & n_{i}<n \leq n_{i+1}\end{array}\right.$, and the sequence $\left\{\phi_{\mathcal{A}_{n}}\left(t_{n}\right)\right\}_{n \in \mathbb{N}}$ does not converge to 1 . Thus, we get the contradiction.

Remark 6. Let $\left(\mathcal{X}, \mathcal{M}_{d}\right)$ be the standard fuzzy metric space induced by the metric space $(\mathcal{X}, d)$. In [17] it was observed that a nested sequence of sets $\left\{\mathcal{A}_{n}\right\}_{n \in \mathbb{N}}$ has fuzzy diameter zero if and only if $\lim _{n} \operatorname{diam}\left(\mathcal{A}_{n}\right)=0$.

In this context, we give the following proposition.

Proposition 4. Let $\left\{\mathcal{A}_{n}\right\}_{n \in \mathbb{N}}$ be a nested sequence of sets with fuzzy diameter zero in $\left(\mathcal{X}, \mathcal{M}_{d}\right) .\left\{\mathcal{A}_{n}\right\}_{n \in \mathbb{N}}$ has strong fuzzy diameter zero if and only if $\mathcal{A}_{n}$ is a singleton set after a certain stage.

Proof. We prove the direct. Suppose $\left\{\mathcal{A}_{n}\right\}_{n \in \mathbb{N}}$ is not eventually constant. Put $s_{n}=\sup \{d(\xi, \eta): \xi, \eta \in$ $\left.\mathcal{A}_{n}\right\}$ and take $t_{n}=s_{n}$ for all $n \in \mathbb{N}$. Then, $\left\{t_{n}\right\}_{n \in \mathbb{N}}$ is a decreasing sequence of positive real numbers which, by Remark 6, converges to 0 . Then,

$$
\begin{aligned}
\lim _{n \rightarrow \infty} \phi_{\mathcal{A}_{n}}\left(t_{n}\right) & =\lim _{n \rightarrow \infty} \inf \left\{\mathcal{M}_{d}\left(\xi, \eta, t_{n}\right): \xi, \eta \in \mathcal{A}_{n}\right\} \\
& =\lim _{n \rightarrow \infty} \frac{t_{n}}{t_{n}+\operatorname{diam}\left(\mathcal{A}_{n}\right)}=\lim _{n \rightarrow \infty} \frac{s_{n}}{s_{n}+s_{n}}=\frac{1}{2}
\end{aligned}
$$

and hence $\left\{\mathcal{A}_{n}\right\}_{n \in \mathbb{N}}$ has not strong fuzzy diameter zero.

The converse is obvious.

Example 1. Let $\mathcal{X}$ be the real interval $[0,+\infty[$. We consider on $\mathcal{X}$ the usual metric $d$, and the induced standard fuzzy metric $\mathcal{M}_{d}$. In addition, we consider on $\mathcal{X}$ the fuzzy metric $\mathcal{M}$ (for the product $t$-norm) given by $\mathcal{M}(\xi, \eta, t)=\frac{\min \{\xi, \eta\}+t}{\max \{\xi, \eta\}+t}$ for each $\xi, \eta \in\left[0,+\infty\left[\right.\right.$ and $t \in \mathbb{R}^{+}$.

Consider also the nested sequences $\left\{\mathcal{A}_{n}\right\}_{n \in \mathbb{N}}$ and $\left\{\mathcal{B}_{n}\right\}_{n \in \mathbb{N}}$ defined by $\mathcal{A}_{n}=\left[0, \frac{1}{n}\right]$ and $\mathcal{B}_{n}=\left[1,1+\frac{1}{n}\right]$, for all $n \in \mathbb{N}$, respectively.

(a) Since $\lim _{n \rightarrow \infty} \operatorname{diam}\left(\mathcal{A}_{n}\right)=\lim _{n \rightarrow \infty} \operatorname{diam}\left(\mathcal{B}_{n}\right)=\lim _{n \rightarrow \infty} \frac{1}{n}=0$ for the metric $d$, then, by Remark $6,\left\{\mathcal{A}_{n}\right\}_{n \in \mathbb{N}}$ and $\left\{\mathcal{B}_{n}\right\}_{n \in \mathbb{N}}$ have fuzzy diameter zero for $\mathcal{M}_{d}$, but they have not strong fuzzy diameter zero for $\mathcal{M}_{d}$ by Proposition 4.

(b) We claim that $\left\{\mathcal{A}_{n}\right\}$ has fuzzy diameter zero in $(\mathcal{X}, \mathcal{M}, \cdot)$. Indeed, take $t \in \mathbb{R}^{+}$, then $\lim _{n \rightarrow \infty} \phi_{\mathcal{A}_{n}}(t)=$ $\lim _{n \rightarrow \infty} \inf \left\{\frac{\min \{\xi, \eta\}+t}{\max \{\xi, \eta\}+t}: \xi, \eta \in \mathcal{A}_{n}\right\}=\lim _{n \rightarrow \infty} \frac{t}{\frac{1}{n}+t}=1$ and so $\left\{\mathcal{A}_{n}\right\}_{n \in \mathbb{N}}$ has fuzzy diameter zero in $(\mathcal{X}, \mathcal{M}, \cdot)$.

We claim that $\left\{\mathcal{A}_{n}\right\}_{n \in \mathbb{N}}$ has not strong fuzzy diameter zero in $(\mathcal{X}, \mathcal{M}, \cdot)$. Indeed, take the decreasing sequence of positive real numbers $\left\{t_{n}\right\}_{n \in \mathbb{N}}$ where $t_{n}=\frac{1}{n}$ for all $n \in \mathbb{N}$. Clearly, $\left\{t_{n}\right\}_{n \in \mathbb{N}}$ converges to 0 . Then,

$$
\lim _{n \rightarrow \infty} \phi_{\mathcal{A}_{n}}\left(t_{n}\right)=\lim _{n \rightarrow \infty} \inf \left\{\frac{\min \{\xi, \eta\}+t_{n}}{\max \{\xi, \eta\}+t_{n}}: \xi, \eta \in \mathcal{A}_{n}\right\}=\lim _{n \rightarrow \infty} \frac{\frac{1}{n}}{\frac{1}{n}+\frac{1}{n}}=\frac{1}{2}
$$

and hence $\left\{\mathcal{A}_{n}\right\}_{n \in \mathbb{N}}$ has not strong fuzzy diameter zero in $(\mathcal{X}, \mathcal{M}, \cdot)$. 
We claim that $\left\{\mathcal{B}_{n}\right\}_{n \in \mathbb{N}}$ has strong fuzzy diameter zero in $(\mathcal{X}, \mathcal{M}, \cdot)$. Indeed, for a decreasing sequence of positive real numbers $\left\{t_{n}\right\}_{n \in \mathbb{N}}$ that converges to 0 we have that

$$
\lim _{n \rightarrow \infty} \phi_{\mathcal{B}_{n}}\left(t_{n}\right)=\lim _{n \rightarrow \infty} \inf \left\{\frac{\min \{\xi, \eta\}+t_{n}}{\max \{\xi, \eta\}+t_{n}}: \xi, \eta \in \mathcal{B}_{n}\right\}=\lim _{n \rightarrow \infty} \frac{1+t_{n}}{1+\frac{1}{n}+t_{n}}=1
$$

and hence $\left\{\mathcal{B}_{n}\right\}_{n \in \mathbb{N}}$ has strong fuzzy diameter zero in $(\mathcal{X}, \mathcal{M}, \cdot)$.

\section{Characterization of Strong Completeness}

We begin this section with the following natural definition.

Definition 9. Let $(\mathcal{X}, \mathcal{M}, *)$ be a fuzzy metric space. $(\mathcal{X}, \mathcal{M}, *)$, or simply $\mathcal{X}$, is called strongly complete if every strong Cauchy sequence in $\mathcal{X}$ is convergent in $\left(\mathcal{X}, \tau_{\mathcal{M}}\right)$.

Since every strong Cauchy sequence is, obviously, Cauchy, we have the following immediate result.

Proposition 5. Every complete fuzzy metric space is strongly complete.

The following is a nice result, in our fuzzy setting.

Proposition 6. Let $\left\{\xi_{n}\right\}_{n \in \mathbb{N}}$ be a sequence in $(\mathcal{X}, \mathcal{M}, *)$. They are equivalent:

(i) $\left\{\xi_{n}\right\}_{n \in \mathbb{N}}$ is convergent and strong Cauchy.

(ii) $\left\{\xi_{n}\right\}_{n \in \mathbb{N}}$ is strongly convergent.

Proof. $(i) \rightarrow$ (ii) Suppose $\left\{\xi_{n}\right\}_{n \in \mathbb{N}}$ is convergent to $\xi$. Let $\left.r \in\right] 0,1[$. We can find $s \in] 0,1[$ such that $(1-s)>1-r$. For $s \in] 0,1\left[\right.$ we can find $n_{s} \in \mathbb{N}$ such that $\mathcal{M}\left(\xi_{m}, \xi_{n}, t\right)>1-s$ for all $m, n \geq n_{s}$ and all $t \in \mathbb{R}^{+}$, since $\left\{\xi_{n}\right\}_{n \in \mathbb{N}}$ is strong Cauchy (notice that $n_{s}$ depends on $r$ since $s$ depends on $r$ ). Now, we have that

$$
\mathcal{M}\left(\xi, \xi_{n}, t\right) \geq \mathcal{M}\left(\xi, \xi_{m}, \frac{t}{2}\right) * \mathcal{M}\left(\xi_{m}, \xi_{n}, \frac{t}{2}\right) \geq \mathcal{M}\left(\xi, \xi_{m}, \frac{t}{2}\right) *(1-s)
$$

for all $m, n \geq n_{s}$ and all $t \in \mathbb{R}^{+}$.

When $m$ tends to $\infty$ we have $\mathcal{M}\left(\xi, \xi_{n}, t\right) \geq 1 *(1-s)>1-r$ for all $n \geq n_{s}$ and all $t \in \mathbb{R}^{+}$, and so $\left\{\xi_{n}\right\}_{n \in \mathbb{N}}$ is strongly convergent to $\xi$.

$($ ii $) \rightarrow(i)$ Suppose that $\left\{\xi_{n}\right\}_{n \in \mathbb{N}}$ is strongly convergent. It is obvious that $\left\{\xi_{n}\right\}_{n \in \mathbb{N}}$ is convergent. On the other hand in [15] (Proposition 4.3), it is proved that $\left\{\xi_{n}\right\}_{n \in \mathbb{N}}$ is strong Cauchy.

Example 2. (A (non-trivial) strongly complete fuzzy metric space).

Let $\mathcal{X}=[0,+\infty[$. It is well-known that $(\mathcal{X}, \mathcal{M}, \cdot)$ is a complete fuzzy metric space where $\mathcal{M}(\xi, \eta, t)=$ $\frac{\min \{\xi, \eta\}+t}{\max \{\xi, \eta\}+t}$ for each $\xi, \eta \in X$ and $t \in \mathbb{R}^{+}$, and that $\tau_{\mathcal{M}}$ is the usual topology of $\mathbb{R}$ restricted to $\mathcal{X}$ [22].

We claim that every strongly convergent sequence to 0 in $\mathcal{X}$ is eventually constant (i.e., the null sequence). Indeed, suppose that $\left\{\xi_{n}\right\}_{n \in \mathbb{N}}$ is strongly convergent to 0 . Given $r=\frac{1}{2}$, there exists $n_{r} \in \mathbb{N}$ such that $\mathcal{M}\left(0, \xi_{n}, t\right)=\frac{t}{\xi_{n}+t}>1-r=\frac{1}{2}$ for all $n \geq n_{r}$ and all $t \in \mathbb{R}^{+}$. Now, if $\left\{\xi_{n}\right\}_{n \in \mathbb{N}}$ is not eventually constant then there exists a subsequence $\left\{\xi_{k_{n}}\right\}_{n \in \mathbb{N}}$ of $\left\{\xi_{n}\right\}_{n \in \mathbb{N}}$, with $\xi_{k_{n}} \neq 0$ for all $n \in \mathbb{N}$, converging to 0 . Take $t_{n}=\frac{1}{2} \cdot \xi_{k_{n}}$ for all $n \in \mathbb{N}$. Then, $\lim _{n \rightarrow \infty} \mathcal{M}\left(0, \xi_{k_{n}}, t_{n}\right)=\lim _{n \rightarrow \infty} \frac{t_{n}}{\xi_{k_{n}}+t_{n}}=\lim _{n \rightarrow \infty} \frac{t_{n}}{2 t_{n}+t_{n}}=\frac{1}{3}$, a contradiction. 
Consider now $\left.\mathcal{X}^{*}=\right] 0,+\infty\left[\right.$ with the fuzzy metric $\mathcal{M}$ restricted to $\mathcal{X}^{*}$. Since $\left\{\frac{1}{n}\right\}_{n \in \mathbb{N}}$ is convergent in $\mathcal{X},\left\{\frac{1}{n}\right\}_{n \in \mathbb{N}}$ is Cauchy in $\mathcal{X}$ and so it is Cauchy in $\mathcal{X}^{*}$. Now, $\left\{\frac{1}{n}\right\}_{n \in \mathbb{N}}$ does not converge in $\mathcal{X}^{*}$ and so $\mathcal{X}^{*}$ is not complete. We show that $\left(\mathcal{X}^{*}, \mathcal{M}, \cdot\right)$ is strongly complete.

Let $\left\{\xi_{n}\right\}_{n \in \mathbb{N}}$ be a non-trivial strong Cauchy sequence in $\mathcal{X}^{*}$. We prove that $\left\{\xi_{n}\right\}_{n \in \mathbb{N}}$ converges in $\left(\mathcal{X}^{*}, \mathcal{M}, \cdot\right)$. Let $\left.r \in\right] 0,1\left[\right.$. Then, we can find $n_{r} \in \mathbb{N}$ such that $\mathcal{M}\left(\xi_{m}, \xi_{n}, t\right)=\frac{\min \left\{\xi_{n}, \xi_{m}\right\}+t}{\max \left\{\xi_{n}, \xi_{m}\right\}+t}>1-r$ for all $m, n \in \mathbb{N}$ with $m, n \geq n_{r}$, and all $t \in \mathbb{R}^{+}$. When $t$ tends to 0 , we have

$$
\lim _{t \rightarrow 0} \mathcal{M}\left(\xi_{m}, \xi_{n}, t\right)=\frac{\min \left\{\xi_{m}, \xi_{n}\right\}}{\max \left\{\xi_{m}, \xi_{n}\right\}} \geq 1-r
$$

for all $m, n \geq n_{r}$, i.e., $\lim _{m, n \rightarrow \infty} \frac{\min \left\{\xi_{m}, \xi_{n}\right\}}{\max \left\{\xi_{m}, \xi_{n}\right\}}=1$, since $r$ is arbitrary.

We claim that $\left\{\xi_{n}\right\}_{n \in \mathbb{N}}$ is upper bounded for the usual metric of $\mathbb{R}$. Indeed, in the other case, there exists a non-bounded increasing subsequence $\left\{\xi_{k_{n}}\right\}_{n \in \mathbb{N}}$ of $\left\{\xi_{n}\right\}_{n \in \mathbb{N}}$ such that $\lim _{n \rightarrow \infty} \frac{\min \left\{\xi_{m}, \xi_{k_{n}}\right\}}{\max \left\{\xi_{m}, \xi_{k_{n}}\right\}}=\lim _{n \rightarrow \infty} \frac{\xi_{m}}{\xi_{k_{n}}}=0$, for all term $\xi_{m}$ of the sequence, a contradiction with (1).

Thus, for some $K>0$, the sequence $\left\{\xi_{n}\right\}_{n \in \mathbb{N}}$ is contained in the (compact) interval $[0, K]$ of $\mathbb{R}$. Suppose $\left\{\xi_{n}\right\}_{n \in \mathbb{N}}$ is not convergent in $\mathcal{X}^{*}$. Then, with an easy argument one can observe that there exist two subsequences $\left\{\xi_{i_{n}}\right\}_{n \in \mathbb{N}}$ and $\left\{\xi_{j_{n}}\right\}_{n \in \mathbb{N}}$ of $\left\{\xi_{n}\right\}_{n \in \mathbb{N}}$, which converge to two distinct points $\xi$ and $\eta$, respectively, in $[0, K]$. We can suppose $\xi<\eta$. Now, $\xi \neq 0$, since in other case, $\left\{\xi_{n}\right\}_{n \in \mathbb{N}}$ is a strong Cauchy sequence and convergent to 0 in $(\mathcal{X}, \mathcal{M}, \cdot)$, and then by Proposition $6\left\{\xi_{n}\right\}_{n \in \mathbb{N}}$ is a non-trivial strongly convergent sequence to 0 , in $(\mathcal{X}, \mathcal{M}, \cdot)$ (a contradiction with the first above paragraph).

Take $\delta>0$ satisfying $\delta<\frac{1}{2} \min \{\xi, \eta-\xi\}$. Then, $] \xi-\delta, \xi+\delta[\cap] \eta-\delta, \eta+\delta[=\varnothing$ and the sequence $\left\{\xi_{i_{n}}\right\}_{n \in \mathbb{N}}$ is eventually in $] \xi-\delta, \xi+\delta\left[\right.$ and $\left\{\xi_{j_{n}}\right\}_{n \in \mathbb{N}}$ is eventually in $] \eta-\delta, \eta+\delta[$. Take $r>0$ such that $r<1-\frac{\xi+\delta}{\eta-\delta}$. By election of $\delta$, we have $\lim _{n \rightarrow \infty} \frac{\min \left\{\xi_{i_{n}}, \eta_{j_{n}}\right\}}{\max \left\{\xi_{i_{n}}, \eta_{j_{n}}\right\}} \leq \frac{\xi+\delta}{\eta-\delta}<1-r$, a contradiction with Equation (1). Hence, $\left\{\xi_{n}\right\}_{n \in \mathbb{N}}$ is convergent in $] 0,+\infty\left[\right.$ and hence $\left(\mathcal{X}^{*}, \mathcal{M}\right)$ is strongly complete.

Finally, we see that there are non-trivial strong Cauchy sequences in $\mathcal{X}^{*}$. Indeed, consider the sequence $\left\{\xi_{n}\right\}_{n \in \mathbb{N}}$ in $\mathcal{X}^{*}$ given by $\xi_{n}=1+\frac{1}{n}$ for all $n \in \mathbb{N}$. Let $\left.r \in\right] 0,1\left[\right.$ and choose $n_{r} \in \mathbb{N}$ satisfying $n_{r}>\frac{1-r}{r}$. Then, $\mathcal{M}\left(1, \xi_{n}, t\right)=\frac{1+t}{1+\frac{1}{n}+t} \geq \frac{1}{1+\frac{1}{n}}>1-r$ for all $n \geq n_{r}$ and all $t \in \mathbb{R}^{+}$. Then, $\left\{\xi_{n}\right\}_{n \in \mathbb{N}}$ is strongly convergent and thus it is strong Cauchy.

Remark 7. In $\left(\mathcal{X}^{*}, \mathcal{M}, \cdot\right)$, every convergent sequence is strongly convergent. Indeed, suppose $\left\{\xi_{n}\right\}_{n \in \mathbb{N}}$ converges to $\xi$ in $\mathcal{X}^{*}$. Given $\left.r \in\right] 0,1\left[\right.$, we choose $\delta>0$ with $\frac{2 \delta}{\xi+\delta}<r$. Now, $\left\{\xi_{n}\right\}_{n \in \mathbb{N}}$ is eventually in ]$\xi-\delta, \xi+\delta\left[\right.$ that is there exists $n_{r}$ such that $\left.\xi_{n} \in\right] \xi-\delta, \xi+\delta\left[\right.$ for all $n \geq n_{r}$, and thus

$$
\mathcal{M}\left(\xi_{,} \xi_{n}, t\right)=\frac{\min \left\{\xi, \xi_{n}\right\}+t}{\max \left\{\xi_{,} \xi_{n}\right\}+t} \geq \frac{\min \left\{\xi, \xi_{n}\right\}}{\max \left\{\xi, \xi_{n}\right\}} \geq \frac{\xi-\delta}{\xi+\delta}>1-r
$$

for all $n \geq n_{r}$ and all $t \in \mathbb{R}^{+}$.

Lemma 1. Let $(\mathcal{X}, \mathcal{M}, *)$ be a fuzzy metric space. If $\left\{\mathcal{A}_{n}\right\}_{n \in \mathbb{N}}$ is a nested sequence of sets of $\mathcal{X}$ which has strong fuzzy diameter zero then $\left\{\overline{\mathcal{A}}_{n}\right\}_{n \in \mathbb{N}}$ has strong fuzzy diameter zero.

Proof. First, we prove that $\phi_{\overline{\mathcal{A}}}(t)=\phi_{\mathcal{A}}(t)$ for every subset $\mathcal{A}$ of $\mathcal{X}$. Indeed, take $\xi, \eta \in \overline{\mathcal{A}}$. Then, we can find two sequences $\left\{\xi_{n}\right\}_{n \in \mathbb{N}}$ and $\left\{\eta_{n}\right\}_{n \in \mathbb{N}}$ in $\mathcal{A}$ that converge to $\xi$ and $\eta$, respectively. Let $t \in \mathbb{R}^{+}$ and an arbitrary $\varepsilon \in] 0,1[$. We have that

$$
M(\xi, \eta, t+2 \varepsilon) \geq M\left(\xi, \xi_{n}, \varepsilon\right) * M\left(\xi_{n}, \eta_{n}, t\right) * M\left(\eta_{n}, \eta, \varepsilon\right) \geq M\left(\xi, \xi_{n}, \varepsilon\right) * \phi_{\mathcal{A}}(t) * M\left(\eta_{n}, \eta, \varepsilon\right)
$$


and taking limit on the inequality when $n$ tends to $\infty$, we obtain

$$
M(\xi, \eta, t+2 \varepsilon) \geq 1 * \phi_{\mathcal{A}}(t) * 1=\phi_{\mathcal{A}}(t)
$$

Since $\varepsilon$ is arbitrary, due to the continuity of $\mathcal{M}\left(\xi, \eta,_{-}\right)$we obtain $\mathcal{M}(\xi, \eta, t) \geq \phi_{\mathcal{A}}(t)$ and then $\phi_{\overline{\mathcal{A}}(t)} \geq \phi_{\mathcal{A}}(t)$. On the other hand, by (ii) of Remark 1 , we have $\phi_{\mathcal{A}}(t) \geq \phi_{\overline{\mathcal{A}}}(t)$ and hence $\phi_{\overline{\mathcal{A}}}(t)=\phi_{\mathcal{A}}(t)$.

Let $\left\{t_{n}\right\}_{n \in \mathbb{N}}$ be a decreasing sequence of positive real numbers converging to 0 . By Proposition 3 we have that $\lim _{n \rightarrow \infty} \phi_{\mathcal{A}_{n}}\left(t_{n}\right)=1$. Then, by our last argument, we have that $\lim _{n \rightarrow \infty} \phi_{\overline{\mathcal{A}}_{n}}\left(t_{n}\right)=\lim _{n \rightarrow \infty} \phi_{\mathcal{A}_{n}}\left(t_{n}\right)=1$, and consequently, by Proposition $3,\left\{\overline{\mathcal{A}}_{n}\right\}_{n \in \mathbb{N}}$ has strong fuzzy diameter zero.

The following is a characterization of the strong completeness by means of nested sequences of closed sets, similar to the completeness of classical metrics.

Theorem 1. Let $(\mathcal{X}, \mathcal{M}, *)$ be a fuzzy metric space. They are equivalent:

(i) $\mathcal{X}$ is strongly complete.

(ii) Every nested sequence of non-empty closed sets $\left\{\mathcal{F}_{n}\right\}_{n \in \mathbb{N}}$ of $\mathcal{X}$ with strong fuzzy diameter zero has a singleton intersection.

Proof. (i) $\rightarrow$ (ii) Suppose $\mathcal{X}$ is strongly complete. Let $\left\{\mathcal{F}_{n}\right\}_{n \in \mathbb{N}}$ be a nested sequence of non-empty closed sets of $\mathcal{X}$ with strong fuzzy diameter zero. Take $\xi_{n} \in \mathcal{F}_{n}$ for each $n \in \mathbb{N}$ and consider the sequence $\left\{\xi_{n}\right\}_{n \in \mathbb{N}}$. Since $\left\{\mathcal{F}_{n}\right\}_{n \in \mathbb{N}}$ has strong fuzzy diameter zero, for a given $\left.r \in\right] 0,1[$ we can find $n_{r} \in \mathbb{N}$ such that $\mathcal{M}(\xi, \eta, t)>1-r$ for all $\xi, \eta \in \mathcal{F}_{n_{r}}$ and all $t \in \mathbb{R}^{+}$. For $m, n \geq n_{r}$ we have that $\xi_{n}, \xi_{m} \in \mathcal{F}_{n_{r}}$ since $\left\{\mathcal{F}_{n}\right\}_{n \in \mathbb{N}}$ is a nested sequence and thus $\mathcal{M}\left(\xi_{n}, \xi_{m}, t\right)>1-r$ for all $n, m \geq n_{r}$ and all $t \in \mathbb{R}^{+}$, and thus $\left\{\xi_{n}\right\}_{n \in \mathbb{N}}$ is a strong Cauchy sequence.

Now, $\mathcal{X}$ is strongly complete and thus $\left\{\xi_{n}\right\}_{n \in \mathbb{N}}$ converges (strongly) to some point, say, $\xi \in \mathcal{X}$. For each fixed $n \in \mathbb{N}$ we have that $\xi_{k} \in \mathcal{F}_{n}$ for all $k \geq n$ and therefore $\xi \in \overline{\mathcal{F}}_{n}=\mathcal{F}_{n}$ for every $n \in \mathbb{N}$ and hence $\xi \in \bigcap_{n=1}^{\infty} \mathcal{F}_{n}$. Finally, $\xi$ is the only element of the intersection. Indeed, suppose $\xi, \eta \in \bigcap_{n=1}^{\infty} \mathcal{F}_{n}$, then since $\left\{\mathcal{F}_{n}\right\}_{n \in \mathbb{N}}$ has (strong) fuzzy diameter zero, for a fixed $t \in \mathbb{R}^{+}$we have $\mathcal{M}(\xi, \eta, t)>1-\frac{1}{n}$ for each $n \in \mathbb{N}$, and thus $\mathcal{M}(\xi, \eta, t)=1$ and hence $\xi=\eta$.

(ii) $\rightarrow(i)$ Let $\left\{\xi_{n}\right\}_{n \in \mathbb{N}}$ be a strong Cauchy sequence in $\mathcal{X}$. We prove that $\left\{\xi_{n}\right\}_{n \in \mathbb{N}}$ is convergent in $\mathcal{X}$ (and according to Proposition 6, $\left\{\xi_{n}\right\}_{n \in \mathbb{N}}$ is strongly convergent). Define $\mathcal{A}_{n}=\left\{\xi_{n}, \xi_{n+1}, \xi_{n+2}, \ldots\right\}$ for all $n \in \mathbb{N}$. For a given $r \in] 0,1\left[\right.$ we can find $n_{r} \in \mathbb{N}$ such that $\mathcal{M}\left(\xi_{m}, \xi_{n}, t\right)>1-r$ for all $m, n \geq n_{r}$ and all $t \in \mathbb{R}^{+}$, since $\left\{\xi_{n}\right\}_{n \in \mathbb{N}}$ is strong Cauchy. Then, $\left\{\mathcal{A}_{n}\right\}_{n \in \mathbb{N}}$ is a nested sequence that has strong fuzzy diameter zero.

For each $n \in \mathbb{N}$ put $\mathcal{F}_{n}=\overline{\mathcal{A}}_{n}$. Then, by Lemma $1,\left\{\mathcal{F}_{n}\right\}_{n \in \mathbb{N}}$ is a nested sequence of non-empty closed sets which has strong fuzzy diameter zero. Hence, by hypothesis, there exists $\xi_{0} \in \mathcal{X}$ such that $\left\{\xi_{0}\right\}=\bigcap_{n=1}^{\infty} \mathcal{F}_{n}$.

Now, for $\varepsilon \in] 0,1\left[\right.$, there exists $n_{\varepsilon} \in \mathbb{N}$ such that $\mathcal{M}(\xi, \eta, t)>1-\varepsilon$ for all $\xi, \eta \in \mathcal{F}_{n}$ with $n \geq n_{\varepsilon}$ and for all $t \in \mathbb{R}^{+}$. In particular, $\mathcal{M}\left(\xi_{0}, \xi_{n}, t\right)>1-\varepsilon$ for all $n \geq n_{\varepsilon}$ and all $t \in \mathbb{R}^{+}$, i.e., $\left\{\xi_{n}\right\}_{n \in \mathbb{N}}$ is (strongly) convergent to $\xi_{0}$, and so $\mathcal{X}$ is strongly complete.

By the last theorem, we obtain the following result for the standard fuzzy metric.

Proposition 7. Let $d$ be a metric on $\mathcal{X}$. Then, the standard fuzzy metric $\left(\mathcal{X}, \mathcal{M}_{d}\right)$ deduced from $d$ is strongly complete. 
Proof. Let $\left\{\mathcal{F}_{n}\right\}_{n \in \mathbb{N}}$ be a nested sequence of sets of $\mathcal{X}$, which has strong fuzzy diameter zero. Then, by Proposition $4,\left\{\mathcal{F}_{n}\right\}_{n \in \mathbb{N}}$ is a singleton set after a certain stage, and, hence, $\bigcap_{n=1}^{\infty} \mathcal{F}_{n}$ is a singleton set. The conclusion follows by Theorem 1 .

Corollary 1. If $(\mathcal{X}, d)$ is a non-complete metric space then $\left(\mathcal{X}, \mathcal{M}_{d}\right)$ is strongly complete and non-complete.

Example 3. We use the last theorem and the completeness of $\mathbb{R}$ with the usual metric of $\mathbb{R}$ to show that the fuzzy metric space (] $0,+\infty[, \mathcal{M}, \cdot)$ of Example 2 is strongly complete.

Let $\left\{\mathcal{F}_{n}\right\}_{n \in \mathbb{N}}$ be a nested sequence of (non-empty) closed subsets of $\left.\mathcal{X}^{*}=\right] 0,+\infty$ [ that has strong fuzzy diameter zero in $\left(\mathcal{X}^{*}, \mathcal{M}, \cdot\right)$. Define $i_{n}=\inf \left\{\xi \in \mathcal{X}^{*}: \xi \in \mathcal{F}_{n}\right\}$ and $s_{n}=\sup \left\{\xi \in \mathcal{X}^{*}: \xi \in \mathcal{F}_{n}\right\}$. Clearly, $i_{n+1} \geq i_{n}$ and $s_{n+1} \leq s_{n}$ for all $n \in \mathbb{N}$, if $s_{n}$ is not infinite. We claim that $\left\{i_{n}\right\}_{n \in \mathbb{N}}$ is not eventually identically zero. Indeed, suppose the contrary, i.e., $i_{n}=0$ for all $n \in \mathbb{N}$. Take $\xi_{n} \in \mathcal{F}_{n}$ for all $n \in \mathbb{N}$ then there exists $\xi_{n}^{\prime} \in \mathcal{F}_{n}$, with $\xi_{n}^{\prime}<\frac{1}{4} \xi_{n}$ for all $n \in \mathbb{N}$. For $r=\frac{1}{2}$ and $t_{n}=\frac{\xi_{n}}{4}$ we have $\mathcal{M}\left(\xi_{n}, \xi_{n}^{\prime}, t_{n}\right)=\frac{\xi_{n}^{\prime}+\frac{\xi_{n}}{4}}{\xi_{n}+\frac{\xi_{n}}{4}}<$ $\frac{\frac{\xi_{n}}{4}+\frac{\xi_{n}}{4}}{\xi_{n}+\frac{\xi_{n}}{4}}=\frac{2}{5}<1-r$ and so $\left\{\mathcal{F}_{n}\right\}_{n \in \mathbb{N}}$ has not strong fuzzy diameter zero.

We claim that $\xi_{n_{0}}<\infty$ for some $n_{0} \in \mathbb{N}$. Indeed, in other case, suppose $s_{n}=\infty$ for all $n \in \mathbb{N}$. Take $\xi_{n} \in \mathcal{F}_{n}$ for all $n \in \mathbb{N}$, then there exists $\xi_{n}^{\prime} \in \mathcal{F}_{n}$ with $4 \xi_{n}<\xi_{n}^{\prime}$ for all $n \in \mathbb{N}$. For $r=\frac{1}{2}$ and $t_{n}=\frac{\xi_{n}}{4}$ we have

$$
\mathcal{M}\left(\xi_{n}, \xi_{n}^{\prime}, t_{n}\right)=\frac{\xi_{n}+\frac{\xi_{n}}{4}}{\xi_{n}^{\prime}+\frac{\xi_{n}}{4}}<\frac{\frac{\xi_{n}}{4}+\frac{\xi_{n}}{4}}{4 \xi_{n}+\frac{\xi_{n}}{4}}=\frac{5}{17}<1-r
$$

and so $\left\{\mathcal{F}_{n}\right\}_{n \in \mathbb{N}}$ has not strong fuzzy diameter zero.

Without loss of generality, we can suppose $i_{1}>0$ and $s_{1}<\infty$. Then, $\mathcal{F}_{n} \subset\left[i_{1}, s_{1}\right]$ for all $n \in \mathbb{N}$, and thus $\left\{\mathcal{F}_{n}\right\}_{n \in \mathbb{N}}$ is a nested sequence of closed sets of $\mathbb{R}$, with the usual topology of $\mathbb{R}$, and so $\bigcap_{n=1}^{\infty} \mathcal{F}_{n} \neq \varnothing$ (this intersection, by Remark 2 , is a unique point), and hence $\left(\mathcal{X}^{*}, \mathcal{M}, \cdot\right)$ is strongly complete.

Denote by $\mathfrak{F}$ (respectively, $\mathfrak{F}_{s t}$ ) a nested sequence of non-empty closed sets on $\mathcal{X}$ that has (respectively, strong) fuzzy diameter zero. Then, taking into account Proposition 5, we have the following nice diagram of implications (of obvious interpretation) for a fuzzy metric space $(\mathcal{X}, \mathcal{M}, *)$.

$$
\begin{array}{cl}
\mathcal{X} \text { complete } & \rightarrow \mathcal{X} \text { strongly complete } \\
\cap & \uparrow \\
\cap \mathfrak{F} \neq \varnothing \text { for all } \mathfrak{F} & \rightarrow \cap \mathfrak{F}_{\text {st }} \neq \varnothing \text { for all } \mathfrak{F}_{\text {st }}
\end{array}
$$

\section{Conclusions}

In this paper, we introduce and study a concept of strong fuzzy diameter zero for a family of sets of a fuzzy metric space $(X, M, *)$, and we characterize when $X$ is strongly complete by means of a certain class of sequences of sets which have strong fuzzy diameter zero (Theorem 1), in a similar way to (classical) metrics.

In our context, several other (well-motivated) concepts of Cauchy sequence have appeared in the literature: standard Cauchy [23], p-Cauchy [24], and s-Cauchy [14] that have been studied in [14]. Then, a natural continuation of this paper is to investigate for which of these concepts one can get a characterization of the corresponding completeness by means of certain classes of nested sequences of sets. On the other hand, the results provided in this document or those above suggested for studying can also be tried to retrieve in the intuitionistic fuzzy context.

Fuzzy fixed point theory, as in the classical case, is founded, basically, on the contractivity of a self-mapping $f$ of $\mathcal{X}$ and the completeness of the fuzzy metric space $\mathcal{X}$. Usually, there are two concepts of completeness extensively used in fuzzy setting. The concept of G-complete space was introduced 
by M. Grabiec [16] and the concept of complete space $[17,25]$ is due to George and Veeramani (a comparative of these two concepts can be seen in [19]). The relationship of these concepts with strong completeness is given in the following chain of implications

$$
G \text { - complete } \rightarrow \text { Complete } \rightarrow \text { Strongly complete }
$$

Then, it arise a natural route of investigation: to state fixed point theorems for strongly complete fuzzy metric spaces. A simple way to proceed is as follows. Suppose an existing theorem $T h$, stated for a complete fuzzy metric space, in the literature. A generalization of $T h$ is reached if one is able to prove that $T h$ is also satisfied when completeness is replaced by strong completeness. To this end, the reader can find fixed point theorems established for complete fuzzy metric spaces in recent publications (see, for instance, [11,26-30] and references therein). Now, this line of investigation is close to the following unsolved question (posed in [15], Problem 4.6): Characterize those fuzzy metric spaces in which Cauchy sequences are strong Cauchy.

Author Contributions: Conceptualization, V.G.; Formal analysis, J.-J.M., B.R. and A.S.; Investigation, V.G., J.-J.M., B.R. and A.S.; Methodology, A.S.; Supervision, V.G., J.-J.M. and A.S.; Writing-original draft, B.R. and A.S.; Writing-review \& editing, J.-J.M. All authors contributed equally in writing this article. All authors have read and agreed to the published version of the manuscript.

Funding: Juan-José Miñana acknowledges financial support from FEDER/Ministerio de Ciencia, Innovación y Universidades-Agencia Estatal de Investigación/_ Proyecto PGC2018-095709-B-C21, and by Spanish Ministry of Economy and Competitiveness under contract DPI2017-86372-C3-3-R (AEI, FEDER, UE). This work was also partially supported by Programa Operatiu FEDER 2014-2020 de les Illes Balears, by project PROCOE/4/2017 (Direcció General d'Innovació i Recerca, Govern de les Illes Balears), and by projects ROBINS and BUGWRIGHT2. These two latest projects have received funding from the European Union's Horizon 2020 research and innovation program under grant agreements Nos. 779776 and 871260, respectively. This publication reflects only the authors views and the European Union is not liable for any use that may be made of the information contained therein.

Conflicts of Interest: The authors declare no conflict of interest.

\section{References}

1. Kramosil, I.; Michalek, J. Fuzzy metric and statistical metric spaces. Kybernetica 1975, 11, 326-334.

2. Menger, K. Statistical metrics. Proc. Natl. Acad. Sci. USA 1942, 28, 535-537. [CrossRef] [PubMed]

3. George, A.; Veeramani, P. On some results in fuzzy metric spaces. Fuzzy Sets Syst. 1994, 64, 395-399. [CrossRef]

4. Schweizer, B.; Sklar, A. Statistical metric spaces. Pac. J. Math. 1960, 10, 314-334.

5. Gregori, V.; Romaguera, S. Some properties of fuzzy metric spaces. Fuzzy Sets Syst. 2000, 115, 485-489. [CrossRef]

6. Gregori, V.; Romaguera, S. On completion of fuzzy metric spaces. Fuzzy Sets Syst. 2002, 130, 399-404. [CrossRef]

7. Atanassov, K.T. Intuitionistic fuzzy sets. Fuzzy Sets Syst. 1986, 20, 87-96. [CrossRef]

8. Gregori, V.; Romaguera, S.; Veeramani, P. A note on intuitionistic fuzzy metric spaces. Chaos Solitons Fractals 2006, 28, 902-905. [CrossRef]

9. Gregori, V.; Sapena, A. Remarks to “on strong intuitionistic fuzzy metrics". J. Nonlinear Sci. Appl. 2018, 11, 316-322. [CrossRef]

10. Abu-Donia, H.M.; Atia, H.A.; Khater Omnia, M.A. Common fixed point theorems in intuitionistic fuzzy metric spaces and intuitionistic $(\varphi, \psi)$-contractive mappings. J. Nonlinear Sci. Appl. 2020, 13, 323-329. [CrossRef]

11. Gregori, V.; Miñana, J.-J. On fuzzy $\psi$-contractive sequences and fixed point theorems. Fuzzy Sets Syst. 2016, 300, 93-101. [CrossRef]

12. Mihet, D. On fuzzy contractive mappings in fuzzy metric spaces. Fuzzy Sets Syst. 2007, 158, 915-921. [CrossRef]

13. Wardowski, D. Fuzzy contractive mappings and fixed points in fuzzy metric spaces. Fuzzy Sets Syst. 2013, 222, 108-114. 
14. Gregori, V.; Miñana, J.-J.; Morillas, S.; Sapena, A. Cauchyness and convergence in fuzzy metric spaces. RACSAM 2017, 11, 25-37.

15. Gregori, V.; Miñana, J.-J. Strong Convergence in Fuzzy Metric Spaces. Filomat 2017, 31, 1619-1625.

16. Grabiec, M. Fixed points in fuzzy metric spaces. Fuzzy Sets Syst. 1988, 27, 385-389.

17. George, A.; Veeramani, P. On some results of analysis for fuzzy metric spaces. Fuzzy Sets Syst. 1997, 90, 365-368.

18. Mihet, D. Fuzzy $\psi$-contractive mappings in non-Archimedean fuzzy metric spaces. Fuzzy Sets Syst. 2008, 159, 739-744. [CrossRef]

19. Vasuki, R.; Veeramani, P. Fixed point theorems and Cauchy sequences in fuzzy metric spaces. Fuzzy Sets Syst. 2003, 135, 415-417. [CrossRef]

20. Gregori, V.; Romaguera, S. Characterizing completable fuzzy metric spaces. Fuzzy Sets Syst. 2004, 144, 411-420.

21. George, A.; Veeramani, P. Some theorems in fuzzy metric spaces. J. Fuzzy Math. 1995, 3, 933-940.

22. Gregori, V.; Miñana, J.-J.; Morillas, S. Some questions in fuzzy metric spaces. Fuzzy Sets Syst. 2012, 204, 71-85. [CrossRef]

23. Ricarte, L.A.; Romaguera, S. A domain-theoretic approach to fuzzy metric spaces. Topol. Appl. 2014, 163, 149-159. [CrossRef]

24. Gregori, V.; López-Crevillén, A.; Morillas, S.; Sapena, A. On convergence in fuzzy metric spaces. Topol. Appl. 2009, 156, 3002-3006. [CrossRef]

25. Sherwood, H. On the completion of probabilistic metric spaces. Zeitschrift für Wahrscheinlichkeitstheorie und Verwandte Gebiete 1966, 6, 62-64.

26. Dinarvand, M. Some fixed point results for admissible Geraghty contraction type mappings in fuzzy metric spaces. Iran. J. Fuzzy Syst. 2017, 14, 161-177.

27. Shukla, S.; Gopal, D.; Sintunavarat, W. A new class of fuzzy contractive mappings and fixed point theorems. Fuzzy Sets Syst. 2018, 350, 85-94.

28. Beg, I.; Gopal, D.; Došenović, T.; Rakić, D. $\alpha$-Type fuzzy $\mathcal{H}$-contractive mappings in fuzzy metric. Fixed Point Theory 2018, 19, 463-474. [CrossRef]

29. Zheng, D.W.; Wang, P. Meir-Keeler theorems in fuzzy metric spaces. Fuzzy Sets Syst. 2019, 370, $120-128$.

30. Rakić, D.; Došenović, T.; Mitrović, Z.D.; de la Sen, M.; Radenović, S. Some Fixed Point Theorems of Cirić Type in Fuzzy Metric Spaces. Mathematics 2020, 8, 297.

(C) 2020 by the authors. Licensee MDPI, Basel, Switzerland. This article is an open access article distributed under the terms and conditions of the Creative Commons Attribution (CC BY) license (http:/ / creativecommons.org/licenses/by/4.0/). 